\title{
Complete Remission of Brain Metastases in Non-Small Cell Lung Cancer Patients Harboring an EGFR Mutation Treated with Tyrosine Kinase Inhibitor without Radiotherapy: A Report of 3 Cases
}

\author{
Yun Hwa Jung Chi Wha Han Yun Duk Jung Young Yun Cho \\ Deok Jae Han \\ Division of Hematology-Oncology, Department of Internal Medicine, \\ Yeouido St. Mary's Hospital, Seoul, Republic of Korea
}

\section{Key Words}

Non-small cell lung cancer · Tyrosine kinase inhibitor · Brain metastases · Whole brain radiotherapy

\section{Abstract}

Brain parenchymal metastasis from a solid tumor is a serious clinical condition associated with a poor outcome because systemic chemotherapy is usually ineffective for treating brain metastases (BM) due to the blood-brain barrier. Therefore, radiotherapy such as whole brain radiotherapy (WBRT) and stereotactic radiosurgery have taken on a central role in the management of BM. However, WBRT can delay subsequent systemic treatment or cause neurologic complications such as a decline in cognitive function. Therefore, suspending WBRT is worth considering if there is an effective alternative. Although there have been no large prospective studies, many reports are available about the favorable effect of tyrosine kinase inhibitors (TKIs) for treating BM in patients with non-small cell lung cancer (NSCLC). Here, we report 3 NSCLC cases that showed a complete response in BM after TKI treatment without WBRT. Based on these remarkable response rates of BM to a TKI, the potential toxicity of WBRT can be avoided, particularly in patients with small metastatic nodules and an epidermal growth factor receptor activating mutation. 
Jung et al.: Complete Remission of BM in NSCLC Patients Treated with EGFR-TKI

\section{Introduction}

Brain metastases (BM) are a relatively frequent but serious complication in patients with non-small cell lung cancer (NSCLC). Although the most reliable methods to control intracranial metastases are surgical resection or stereotactic radiosurgery, whole brain radiotherapy (WBRT) is considered the standard option for cases of multiple metastases or out of concern for invisible metastasis. However, possible late neurologic sequelae of WBRT make physicians anxious. Therefore, more customized treatment strategies for BM are required in order to avoid late complications of WBRT. Epidermal growth factor receptortyrosine kinase inhibitors (EGFR-TKIs) have shown remarkable response rates for intracranial lesions as well as extracranial lesions in NSCLC patients harboring EGFR activating mutations. Here, we report 3 cases that showed complete regression of intracranial lesions after treatment with only gefitinib, without WBRT. Furthermore, we summarize previous studies supporting a treatment strategy that uses an upfront EGFR-TKI without radiotherapy.

\section{Case Report}

Case 1

A 60-year-old man with a history of smoking presented at our hospital due to back pain and a chest X-ray abnormality. Lung adenocarcinoma was diagnosed with a bronchoscopic biopsy, and whole body positron emission tomography-computed tomography (PET-CT) showed multiple metastases on the lumbar spine and pelvic bone. The tumor was found to have an EGFR mutation (exon 19 deletion) and sequential brain magnetic resonance imaging (MRI) revealed multiple tiny enhancing nodules in the left occipital lobe and cerebellum. Gefitinib $(250 \mathrm{mg} /$ day) was administered and radiotherapy was performed on the lumbar vertebra. WBRT was deferred due to the absence of symptoms. One month after gefitinib treatment was initiated, interval regression of BM was identified on a follow-up brain MRI. After another 3 months, metastatic lesions were not discernible on a follow-up brain MRI (fig. 1a).

Case 2

A 72-year-old woman without a history of smoking presented with supraclavicular lymphadenopathy. An incisional biopsy of the lymph node was performed, and the pathologic result was adenocarcinoma with cytokeratin-7 (CK-7) and thyroid transcription factor-1 (TTF-1) positivity on immunohistochemical staining. The tumor was harboring an EGFR mutation (exon 19 deletion). Whole body PET-CT showed multiple bone metastases with mediastinal lymphadenopathy. Brain MRI identified well-defined enhancing round nodules on the right frontal and left parietal lobes. She received gefitinib (250 mg/day) and palliative radiotherapy for the painful pelvic bone metastases. WBRT was put off because she did not complain of neurologic symptoms. A brain MRI performed 6 months later showed interval regression of multiple BM. The metastatic nodules disappeared after another 3 months (fig. 1b).

Case 3

A 74-year-old woman without a history of smoking presented with a scalp mass. An incisional biopsy performed on the scalp mass revealed adenocarcinoma with CK-7 and TTF1 positivity on immunohistochemical staining. An EGFR mutation analysis found an exon 21 
L858R point mutation. Whole body PET-CT detected a lung mass and multiple bone metastases. Brain MRI identified several tiny enhancing nodules on both cerebral hemispheres. After 5 months of gefitinib treatment ( $250 \mathrm{mg} /$ day), a follow-up brain MRI showed no brain parenchymal metastatic lesions (fig. 1c).

\section{Discussion}

BM are a relatively frequent and grave complication of NSCLC. Considering prolonged overall survival results from superior systemic treatment, the incidence of BM is expected to rise. Therefore, treatment strategies for BM need to commensurate with the development of systemic treatments. Because of the marginal effect of systemic chemotherapy, radiotherapy or surgery has been considered a standard treatment for BM from lung cancer. However, encouraging intracranial effects of some systemic chemotherapeutic agents, including EGFRTKIs, have been gradually proposed [1]. In particular, in a few case reports and retrospective/prospective studies [2-4], outstanding effects of EGFR-TKIs on BM have been reported in patients harboring EGFR activating mutations. Considering that NSCLC is a relatively radioresistant malignancy and the possibility of late neurologic sequelae of WBRT, EGFRTKIs are considered a promising option on central nervous system metastases from lung adenocarcinoma. However, there is no consensus on an optimal TKI agent, the reliability of EGFR mutation status of the primary tumor as a surrogate marker of EGFR mutation status of BM, temporal arrangement of WBRT and EGFR-TKI treatment or management of intracranial local failure after TKI treatment [5]. Among them, substitution of WBRT with an EGFR-TKI as an initial treatment for BM has been a subject of concern.

Firstly, it has been proposed that EGFR-TKIs should be administered as concomitant agents with WBRT for BM in patients with NSCLC because blocking EGFR signaling in vitro has been known to sensitize cancer cells to the effects of radiation. In practice, Zeng et al. [6] compared the efficacy of gefitinib alone with gefitinib plus concomitant WBRT and insisted that concurrent treatment improves response rate and overall survival. However, the EGFR mutation status of the enrolled patients was not reported. Moreover, the response rate in the patient group treated with concomitant gefitinib and WBRT was not significantly higher than that of previously reported studies which targeted only patients harboring EGFR mutations treated with EGFR-TKI alone. A few subsequent studies including erlotinib also showed similar results $[7,8]$. Therefore, the additional benefit of concomitant radiation is uncertain, particularly in NSCLC patients harboring EGFR activating mutations.

Furthermore, we searched for and summarized studies regarding the intracranial response rate of EGFR-TKIs with or without WBRT in lung adenocarcinoma (table 1). Based on our summary, the response rate to EGFR-TKI only treatment was comparable to that of EGFR-TKI plus WBRT concomitant therapy. Central nervous system failure interval of the concurrent treatment group was not longer than that of the EGFR-TKI only treatment group. However, we should consider the potential harmful effect of WBRT on neurologic function. Assessing impairment in neurocognitive function in patients treated with WBRT is difficult, and to estimate net effect of WBRT on neurocognitive function, many variables such as other oncological treatments, including chemotherapy, anticonvulsants, opioids and neurocognitive dysfunction caused by metastases itself, should be considered. Therefore, controversy remains about how significant the detrimental effect of WBRT is on neurocognitive function.

However, many studies supported these concerns with reasonable evidence [9]. In particular, 1 study aimed at patients without BM who received prophylactic cranial irradiation showed a significant decline in neurocognitive function compared with that in 
the observation group [10]. Along with those results, various efforts such as focal therapyoriented strategy, dose per fraction reduction for prophylactic cranial irradiation and hippocampal-sparing radiotherapy are being discussed to avoid or reduce late complications of WBRT [11]. Therefore, there is no reason that upfront WBRT should be performed at the risk neurologic complications if there are effective alternatives. Some oncologists justify upfront WBRT for several reasons, even in patients harboring EGFR activating mutations. Hirata et al. [12] reported that acquired resistance to TKIs triggers cross-resistance to radiotherapy, whereas TKI-naive patients with an EGFR mutation exhibit better response to brain radiotherapy than those with wild-type EGFR. However, continuing TKI after progression, increasing the dose of TKI or switching to a second-generation TKI after acquired resistance to a TKI can be considered a solution for diminished radiosensitivity $[13,14]$.

In addition, other clinical indicators predicting a favorable intracranial response to TKIs except EGFR mutations should be investigated to select appropriate patients who can avoid upfront WBRT. Although there are no statistical data, BM size is thought to be an important predictive factor. Based on previous reports that analyzed TKI concentration in cerebrospinal fluid, TKIs were not free from the blood-brain barrier either. That is, the blood-brain barrier acts as an obstacle for TKIs to penetrate the brain parenchyma [15]. Consequently, the size of the metastasis is thought to be affected by the concentration of TKIs and acts as a predictive factor of intracranial response. In that sense, remarkable intracranial control in our cases was also attributed to the small size of the metastases.

In conclusion, BM is not an uncommon complication in patients with NSCLC. A stratified treatment approach is required to balance optimal intracranial disease control and neurologic complications. EGFR-TKI treatment and withholding WBRT is a feasible option in asymptomatic patients with small metastatic nodules harboring an EGFR mutation.

\section{Disclosure Statement}

The authors declare that they have no competing interests.

\section{References}

1 Schwer AL, Gaspar LE: Update in the treatment of brain metastases from lung cancer. Clin Lung Cancer 2006;8:180-186

2 Kim JE, Lee DH, Choi Y, et al: Epidermal growth factor receptor tyrosine kinase inhibitors as a first-line therapy for never-smokers with adenocarcinoma of the lung having asymptomatic synchronous brain metastasis. Lung Cancer 2009;65:351-354.

-3 Muller F, Riesenberg H, Hirmle P, Gehl HB, Duwel P, Gorner M: Complete remission of multiple brain metastases of non-small cell lung cancer induced by gefitinib monotherapy. Strahlenther Onkol 2011;187:826-830.

-4 Park SJ, Kim HT, Lee DH, et al: Efficacy of epidermal growth factor receptor tyrosine kinase inhibitors for brain metastasis in non-small cell lung cancer patients harboring either exon 19 or 21 mutation. Lung Cancer 2012;77:556-560.

5 Berger LA, Riesenberg H, Bokemeyer C, Atanackovic D: CNS metastases in non-small-cell lung cancer: current role of EGFR-TKI therapy and future perspectives. Lung Cancer 2013;80:242-248.

6 Zeng YD, Zhang L, Liao H, et al: Gefitinib alone or with concomitant whole brain radiotherapy for patients with brain metastasis from non-small-cell-lung cancer: a retrospective study. Asian Pac J Cancer Prev 2012;13:909-914.

7 Iuchi T, Shingyoji M, Sakaida T, et al: Phase II trial of gefitinib alone without radiation therapy for Japanese patients with brain metastases from EGFR mutant lung adenocarcinoma. Lung Cancer 2013;82:282-287.

-8 Welsh JW, Komaki R, Amini A, et al: Phase II trial of erlotinib plus concurrent whole-brain radiation therapy for patients with brain metastases from non-small cell lung cancer. J Clin Oncol 2013;31:895-902. 


\section{Case Reports in Oncology}

\begin{tabular}{l|l}
\hline Case Rep Oncol 2014;7:149-154 & \multicolumn{2}{l}{} \\
\hline DOI: $10.1159 / 000360731$ & $\begin{array}{l}\text { c) 2014 S. Karger AG, Basel } \\
\text { www.karger.com/cro }\end{array}$ \\
\hline
\end{tabular}

Jung et al.: Complete Remission of BM in NSCLC Patients Treated with EGFR-TKI

9 McDuff SG, Taich ZJ, Lawson JD, et al: Neurocognitive assessment following whole brain radiation therapy and radiosurgery for patients with cerebral metastases. J Neurol Neurosurg Psychiatry 2013;84:1384-1391.

10 Sun A, Bae K, Gore EM, et al: Phase III trial of prophylactic cranial irradiation compared with observation in patients with locally advanced non-small-cell lung cancer: Neurocognitive and quality-of-life analysis. J Clin Oncol 2011;29:279-286.

11 Shar MG, Ball DL: Treatment of brain metastases in lung cancer: strategies to avoid/reduce late complications of whole brain radiation therapy. Curr Treat Options Oncol 2013;14:553-567.

$\checkmark 12$ Hirata H, Nakamura K, Kunitake N, et al: Association between EGFR-TKI resistance and efficacy of radiotherapy for brain metastases from EGFR-mutant lung adenocarcinoma. Anticancer Res 2013;33: 1649-1655.

13 Katayama T, Shimizu J, Suda K, et al: Efficacy of erlotinib for brain and leptomeningeal metastases in patients with lung adenocarcinoma who showed initial good response to gefitinib. J Thorac Oncol 2009;11:1415-1419.

14 Yu Ha, Sima CS, Huana J, et al: Local therapy with continued EGFR tyrosine kinase inhibitor therapy as a treatment strategy in EGFR-mutant advanced lung cancers that have developed acquired resistance to EGFR tyrosine kinase inhibitors. J Thorac Oncol 2013;8:346-351.

15 Zhao J, Chen M, Zhong W, et al: Cerebrospinal fluid concentrations of gefitinib in patients with lung adenocarcinoma. Clin Lung Cancer 2013;14:188-193.

16 Zhuang H, Yuan Z, Wang J, Zhao L, Pang Q, Wang P: Phase II study of whole brain radiotherapy with or without erlotinib in patients with multiple brain metastases from lung adenocarcinoma. Drug Des Devel Ther 2013;8:1179-1186.

Table 1. Reports about intracranial response to EGFR-TKIs without WBRT in lung cancer patients (adenocarcinoma)

\begin{tabular}{|c|c|c|c|c|c|c|}
\hline Author & $\mathrm{Pt}$ & TKI agent used & WBRT & $\begin{array}{l}\text { Intracranial } \\
\text { response rate, } \\
\%\end{array}$ & $\begin{array}{l}\text { CNS failure } \\
\text { interval, ms }\end{array}$ & EGFR mutation \\
\hline Kim et al. (2009) [2] & 23 & $\begin{array}{l}\text { gefitinib } 250 \mathrm{mg}(\mathrm{n}=16) \text { or } \\
\text { erlotinib } 150 \mathrm{mg}(\mathrm{n}=7)\end{array}$ & ND & 73.9 & 19.3 & confirmed (Y) \\
\hline Park et al. (2012) [4] & 28 & $\begin{array}{l}\text { gefitinib } 250 \mathrm{mg}(\mathrm{n}=22) \text { or } \\
\text { erlotinib } 150 \mathrm{mg}(\mathrm{n}=6)\end{array}$ & ND & 83 & 12.6 & confirmed $(\mathrm{Y})$ \\
\hline Iuchi et al. (2013) [7] & 41 & gefitinib $250 \mathrm{mg}$ & ND & 87.8 & 14.5 & confirmed (Y) \\
\hline Zeng et al. (2012) [6] & 90 & gefitinib $250 \mathrm{mg}$ & $\begin{array}{l}\text { done }(\mathrm{n}=45)(40 \mathrm{~Gy}) \\
\mathrm{ND}(\mathrm{n}=45)\end{array}$ & $\begin{array}{l}\text { done: } 64.4 \\
\text { ND: } 26.7\end{array}$ & $\begin{array}{l}\text { done: } 10.6 \\
\text { ND: } 6.57\end{array}$ & unconfirmed \\
\hline Welsh et al. (2013) [8] & 40 & erlotinib $150 \mathrm{mg}$ & done (35 Gy) & 86 & 8.0 & $\begin{array}{l}Y(n=8) \\
N(n=9) \\
\text { unconfirmed } \\
(n=23)\end{array}$ \\
\hline Zhuang et al. (2013) [16] & 23 & erlotinib $150 \mathrm{mg}$ & done (30 Gy) & 95.65 & $\begin{array}{l}Y: 11.2 \\
\text { N: } 9.6\end{array}$ & $\begin{array}{l}Y(n=11) \\
N(n=12)\end{array}$ \\
\hline
\end{tabular}

$\mathrm{Pt}=$ Number of patients; $\mathrm{Y}=$ yes; $\mathrm{N}=$ no; ND = not done. 


\section{Case Reports in Oncology}
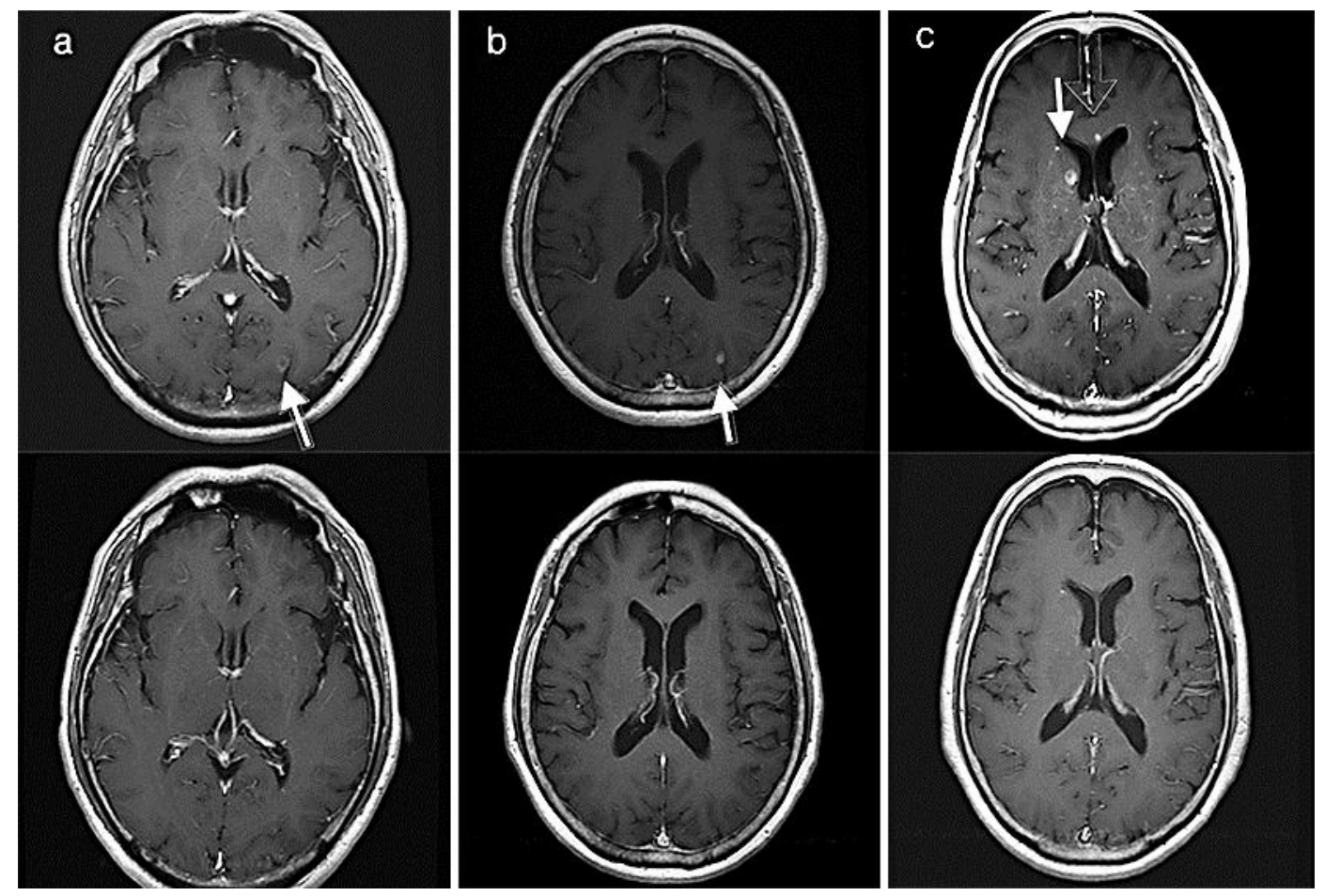

Fig. 1. Brain MRI (a-c) of 3 NSCLC patients before (top row) and after (bottom row) gefitinib treatment. a On contrast enhanced T1 weighted image, a small peripheral enhancing nodule in the left occipital lobe is identified (arrow). b A small, well-defined enhancing round nodule in the left occipital lobe is identified (arrow). c A small enhancing nodule in the right periventricular deep white matter is identified (arrow). 